\title{
Itraconazole Dispersion In Polymer Matrix
}

National Cancer Institute

\section{Source}

National Cancer Institute. Itraconazole Dispersion In Polymer Matrix. NCI Thesaurus. Code C104048.

A proprietary oral formulation composed of the poorly soluble, synthetic triazole agent, itraconazole, dispersed in a polymer matrix, with antifung al and potential anti-angiog enic activities. Upon oral administration, itraconazole inhibits the enzyme cytochrome P450 lanosterol 14 alpha-demethylase, resulting in a decrease in fungal ergosterol synthesis. Although the exact mechanism through which itraconazole inhibits angiogenesis has yet to be fully elucidated, this agent appears to inhibit the Hedgehog $(\mathrm{Hh})$ signaling pathway, cholesterol synthesis and mammalian target of rapamycin (mTOR) signaling in endothelial cells. This agent may also prevent the activation of and signaling by various angiog enic growth factors. By decreasing the tumor vasculature and nutrient supply, itraconazole ultimately inhibits tumor cell growth. The solid dispersion of itraconazole in the polymer matrix enhances dissolution of itraconazole in the gastrointestinal tract and increases its bioavailability; this allows for the administration of a much lower dose compared to itraconazole alone. 